\title{
Concept of Minimum State Density in the Activated Complex Theory of Bimolecular Reactions*
}

\author{
W. H. Wong $\dagger$ and R. A. Marcus \\ Noyes Chemical Laboratory, University of Illinois, Urbana, Illinois 61801
}

(Received 5 August 1971)

\begin{abstract}
In some bimolecular reactions, for example, certain low potential-energy barrier reactions, the saddlepoint choice for the position of the activated complex is vague and sometimes incorrect. In these and in some others a different choice is needed. The concept of minimum state density for this purpose, its relation to adiabatic transition state theory and to the maximum free energy criterion, are discussed. It is shown how it may be applied to bimolecular reactions using microcanonical activated complex theory for these reactions.
\end{abstract}

\section{INTRODUCTION}

Some time ago, microcanonical activated complex theory for bimolecular reactions was described by one of us, ${ }^{1}$ and the resulting expressions have been applied in a number of problems. ${ }^{2-5}$ A recent series of numerical tests $^{1,4-6}$ of activated complex theory using Monte Carlo calculations and exact classical mechanical trajectories make use of the expression or, in the collinear collision case, of its one-dimensional analog. ${ }^{4}$ One problem which arises in activated complex theory in general and of low activation energy reactions in particular concerns the position of the activated complex along the reaction coordinate. In the present paper this position is discussed for bimolecular reactions, both for use in the Monte Carlo tests and for potential application to experimental rate constants.

When the potential-energy barrier for a reaction is sufficiently high and the saddle point on the potentialenergy surface is sufficiently well defined, the position of the activated complex is clear: It is usual saddlepoint choice. In calculations of rate constants for other surfaces the position of maximum local free energy of the system along the reaction coordinate has been suggested for choosing the activated complex. ${ }^{7}$ In the case of a microcanonical ensemble, on the other hand, in a theoretical study testing RRKM unimolecular reaction rate theory using Monte Carlo trajectory data, Bunker and Pattengill ${ }^{8}$ proposed a minimum state density criterion for choosing the position of the activated complex.

We first consider, in Sec. II, a relation between the minimum state density criterion and "adiabatic"9,10 transition state theory. In Secs. III and IV we show how the minimum energy density criterion can be used in numerical tests of bimolecular activated complex theory using microcanonical ensembles and Monte Carlo trajectory data. The use of different subsets of this ensemble (e.g., with constant total angular momentum ${ }^{11}$ ) for certain tests is also noted. Using the $\mathrm{Cl}+\mathrm{HI} \rightarrow \mathrm{HCl}+\mathrm{I}$ reaction, some of the pitfalls which would arise in some cases if consideration were re- stricted to a harmonic-oscillator approximation, are illustrated.

In the concluding section the relation between the minimum state density criterion for microcanonical ensembles and the maximum free energy criterion ${ }^{7}$ for canonical ensembles is described. In applications to experiment, the latter is somewhat simpler to use, but in some recent detailed tests of various aspects of activated complex theory with trajectory data the microcanonical level of detail has been investigated.

\section{RELATION OF MINIMUM STATE DENSITY TO ADIABATIC TRANSITION STATE THEORY}

In reactions having low activation energy the position of the activated complex along the reaction coordinate $s$ is not as obvious as it is for those of higher activation energy. In the latter it is at the top of a potentialenergy barrier. In the former the "bottleneck" for the quasiequilibrium flow of systems from a configuration of reactants to one of products may occur elsewhere as a result of dynamical or "entropic" factors.

Regarding the use of an effective rather than actual potential-energy barrier it is useful to recall first "adiabatic" transition state theory. ${ }^{9,10}$ In that theory each quantum state $n$ of the system is associated with its own effective barrier (Fig. 1). The barrier occurs at some $s, s^{+}(n)$, and is the maximum of the adiabatic potential-energy curve for motion along $s$ for the quantum state. The energy spacing of these curves for adjacent quantum states $n$ varies with $s$, since the local vibration frequencies and moments of inertia for the vibrational and rotational degrees of freedom of the system vary with $s$. Thus, $s^{+}(n)$ will vary with $n$ and need not be close to the position of the maximum of the actual potential-energy maximum, particularly when that barrier is low (Fig. 1).

To incorporate such effects one approach would be to use the adiabatic transition state theor $y^{9,10,12}$ for every single state and sum the contribution from each state to the reaction rate or ensemble-averaged crosssection. However, in many-dimensional systems this 


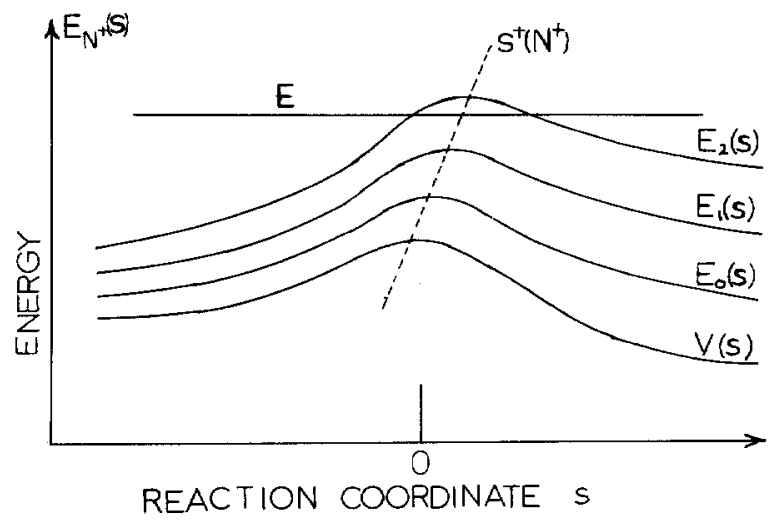

FIG. 1. Schematic plot of energy $E_{N^{+}}(s)$ of adiabatic states vs reaction coordinate. Dotted line indicates position of maximum.

procedure would be cumbersome. (The procedure could not in any event be used to calculate individual cross sections for various quantum states, because deviations from adiabaticity affect individual more than ensembleaveraged cross sections.)

Instead, a second approach would be to find some $s^{+}(n)$ suitably averaged over all states $n$ in the ensemble and use this $\left\langle s^{+}(n)\right\rangle$ to define the position of the transition state. We shall do so in the present paper, utilizing an expression for a microcanonical bimolecular activated complex theory. ${ }^{1}$

The equation for this mocrocanonical ensemble is given by ${ }^{1}$

$$
\sum_{N}\left(k^{2} / \pi\right) \sigma_{N_{p}}=\sum_{N^{+}} \kappa\left(E, N^{+}\right),
$$

where $N$ and $\mathrm{N}^{+}$denote an internal quantum state of the reacting pair and of the activated complex, respectively. $\sigma_{N p}$ is the reaction cross section of a pair reacting from state $N$ and having initial relative translational momentum $p ; \kappa\left(E, N^{+}\right)$is the probability of passage over or through the barrier for a system in state $N^{+}$in the vicinity of the activated complex region; $k$, a wavenumber, equals $p / \hbar$; and the total energy $E$ of the reactants is

$$
E=E_{p}+E_{N}=\left(k^{2} \hbar^{2} / 2 \mu\right)+E_{N},
$$

where $E_{p}$ and $E_{N}$ are the initial relative translational energy $\left(p^{2} / 2 \mu\right)$ and the initial rotational-vibrational energy of the reactants, respectively.

Ordinary (i.e., canonical) activated complex theory is obtained by multiplying both sides of (1) by a Boltzmann factor, $[\exp (-E / k T)] d E / h$, integrating over all $E$, and dividing by $Q$, the partition function of the reactants. The left-hand side then becomes the usual rate constant and the right-hand side becomes, on interchanging order of summation and integration, a "generalized activated complex theory" expression for it, namely that in Ref. 9, Eq. (14). For brevity we have omitted from Eq. (1) and from the present dis- cussion a reaction path degeneracy operator $\Gamma$ present in Eq. (4) of Ref. 1 and in Ref. 9. It offers no difficulty and can also be made to allow for competitive paths. ${ }^{13}$

A natural choice of the activated complex would be one which accurately treats the $\kappa$ terms in a simple way, for example by replacing the right-hand side by some sum over states $N^{+}, \Sigma_{N^{+}}$, at a given value of $s, s^{+}$:

$$
\sum_{N^{+}} \kappa\left(E_{N}, N^{+}\right) \cong \sum_{N^{+},\left(E_{N^{+}} \leq E^{\prime}\right)} 1 \text { at some } s^{+},
$$

where $E_{N^{+}}$is the energy of state $N^{+}$at the given $s^{+}$. There are many more states in the sum on the left side than those on the right, since there are many states on left for which $\kappa$ is negligible. Here, we may refer again to Fig. 1 which describes the energy $E_{N^{+}}(s)$ of various adiabatic states $\mathrm{N}^{+}$along the reaction coordinate $s$. Clearly, too large a negative choice for $s$ (too large a separation distance of reactants) yields many terms for which $\kappa$ would be zero and so is not acceptable. Too large a positive choice for $s$ would again include many states not accessible from the left, since $\kappa$ would again be too small.

The states for which the maximum of the $E_{N^{+}} \max$ imum is below $E$ have $\kappa=1$ when diffraction effects are neglected near the top of each effective barrier. In deciding which $s^{+}$to introduce into (3) we may consider the density of states $N^{+}$in the vicinity of $E_{N} \cong E$. Too negative or too positive an $s$ yields, at least for several-dimensional systems, too large a density of states. (This density increases rapidly with excess energy for such systems.) Thus, an $s$ is picked which minimizes this density, i.e., for which the derivative of the density with respect to $s$ is zero.

This criterion was used by Bunker and Pattengill ${ }^{8}$ in their theoretical study of the RRKM theory of unimolecular decomposition. The latter theory of unimolecular reactions makes use of a microcanonical ensemble. The microcanonical form of bimolecular activated complex theory, described by Eq. (1), permits us to explore this criterion for bimolecular reactions also. The preceding discussion describes a relationship between this criterion and an adiabatic activated complex theory.

\section{THEORY}

One may define, for brevity of notation, an ensembleaveraged cross section at a given energy, $\sigma(E)$,

$$
\sigma(E)=\frac{\sum_{N}\left(k^{2} / \pi\right) \sigma_{N p}}{\sum_{N}\left(k^{2} / \pi\right)},
$$

where the sums are over all states for which $E_{N} \leq E$. [This function is the same as the one denoted by $\bar{S}_{r}(E)$ in Ref. 4.] From Eqs. (1)-(4) we then have

$$
\sigma(E)=\frac{\mathfrak{\varkappa}^{+}(E)}{\sum_{N}\left(k^{2} / \pi\right)},
$$


where

$$
\Re^{+}(E)=\sum_{N^{+},\left(E_{N}+\leq E\right)} 1 .
$$

That is, $\Re^{+}(E)$ is the number of states of the activated complex with rotational-vibrational energy equal to or less than $E$. The other sum in Eq. (5) can be written as an integral using (2), since the trajectory data with which comparison will be made were classical,

$$
\sum_{N} \frac{k^{2}}{\pi}=\frac{2 \mu}{\pi \hbar^{2}} \int_{E_{N}=0}^{E}\left(E-E_{N}\right)\left[\frac{d \Re\left(E_{N}\right)}{d E_{N}}\right] d E_{N}
$$

where $\mathfrak{R}\left(E_{N}\right)$ is the number of rotational-vibrational states of the reactants with initial rotational-vibrational energy equal to or less than $E_{N}$.

The numbers $\mathfrak{\Re}^{+}(E)$ and $\mathscr{N}\left(E_{N}\right)$ are readily obtained by various methods, allowing for or neglecting anharmonicity and formulated by various authors for use with RRKM unimolecular reaction rate theory. ${ }^{14} \mathrm{Sev}$ eral formulas are collected in Appendix A, by way of illustration.

Using the minimum state density criterion for the position of the activated complex, $s^{+}$is that $s$ for which

$$
(\partial / \partial s)\left[d \mathscr{C}^{+}(E) / d E\right]=0 \quad\left(s=s^{+}\right) .
$$

For $s$ one might use the "reaction path" (path of minimum potential energy) discussed by Johnston. ${ }^{15}$

In some reactions the centrifugal potential becomes a significant quantity determining the position of the activated complex (e.g., as in loose activated complexes) and, for such reactions, has to be taken into account in computing $\mathfrak{N}^{+}(E)$. In this case one can use Eq. (5) or (6) of Ref. 1 instead of Eq. (3) there. The centrifugal potential played a role in the unimolecular study of Refs. 8 and 16, for example.

In the next section we give for concreteness, a specific example of Eqs. (4)-(8) for the reaction of an atom with a diatomic molecule, using for ease of presentation a harmonic-oscillator approximation for the vibrations of the molecule and of the activated complex.

\section{REACTION OF AN ATOM AND A DIATOMIC MOLECULE}

When the diatomic molecule is treated as a rigid rotor-harmonic oscillator and when the activated complex is linear and treated as having one symmetric stretching and one doubly degenerate bending vibration (harmonic oscillators) and as being a rigid rotor, the equations in the Appendix yield

$$
\begin{aligned}
\sum_{N} \frac{k^{2}}{\pi} & =\frac{\left(2 \mu / \pi \hbar^{2}\right)\left(8 \pi^{2} I / h^{2}\right) E^{3}}{(3 ! h \nu)_{\sigma}}, \\
\mathfrak{N}^{+}(E) & =\frac{\left(8 \pi^{2} I^{+} / h^{2}\right)\left(E-V^{+}\right)^{4}}{4 !\left(h \nu_{S^{+}}\right)\left(h \nu_{B}+\right)^{2} \sigma^{+}},
\end{aligned}
$$

where $I, \nu$, and $\sigma$ are the moment of inertia, vibration frequency and symmetry number of the diatomic reactant; $V^{+}, I^{+}$, and $\sigma^{+}$are the potential energy, moment of inertia, and symmetry number for the activated complex at the given $s^{+} ; \nu_{S}{ }^{+}$and $\nu_{B}{ }^{+}$are the symmetric stretching and the bending frequencies of the activated complex at that $s$.

When needed one can operate on (10) with the reaction path degeneracy operator $\Gamma$ referred to earlier. ${ }^{1}$

The density of states $d \mathscr{Y}^{+}(E) / d E$ is found from (10) by differentiation with respect to $E$, and this density can be calculated as a function of $s$. A normal mode analysis can be made along the reaction path to obtain the local values of $V^{+}, \nu_{S}^{+}, \nu_{B}{ }^{+}$, and $I^{+}$.

Recently, extensive trajectory studies on the reaction $\mathrm{Cl}+\mathrm{HI} \rightarrow \mathrm{HCl}+\mathrm{I}$ have been made by Polanyi and Wong. ${ }^{17}$ From these studies $\sigma(E)$ is about $7 \AA^{2}$ at a total translational-vibrational-rotational energy $E$ of $5 \mathrm{kcal} \mathrm{mole}{ }^{-1}$ (the $E$ of thermal interest) and about

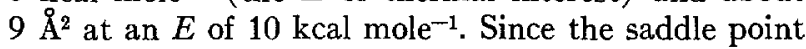
on this surface occurred at a separation distance of reactants of about $4.7 \AA$, the "hard sphere" $\sigma(E)$ that would be calculated if there were no steric restrictions would be $\pi(4.7)^{2}$ or about $70 \AA^{2}$. (The potential-energy barrier for this surface is only $0.1 \mathrm{kcal} \mathrm{mole}{ }^{-1}$.)

As an example of the errors that can occur if the harmonic-oscillator approximation were employed for the very low bending frequencies that prevail for $\mathrm{Cl} \cdot \mathrm{H} \cdot \mathrm{I} \ddagger$ for this surface the $\sigma(E)$ calculated from Eqs. (4), (9), and (10) for a saddle-point configuration is about $210 \AA^{2}$ at an $E$ of $5 \mathrm{kcal} \mathrm{mole}^{-1}$. It is reduced to about $20 \AA^{2}$ when the minimum state density criterion is used (for then $\nu_{B}{ }^{+}$is higher), and one could expect further reductions if the correct strongly anharmonic density of states were used in $\mathfrak{\Re}^{+}(E)$. An example of the use of the latter density appears in Appendix II of Ref. 2(b).

\section{RELATION BETWEEN MINIMUM STATE DENSITY AND MAXIMUM FREE ENERGY CRITERIA}

We comment finally on the relation between the minimum energy density criterion and the maximum free energy criterion for the position of the activated complex.

The reaction rate constant $k_{r}$ equals ${ }^{10}$

$$
k_{r}=\int_{E=0}^{\infty} \sum_{N} \frac{k^{2} \sigma_{N p}}{\pi} \frac{\exp \left(-E / k_{B} T\right) d E}{h Q},
$$

where $Q$ is the partition function of the reactants and $k_{B}$ is the Boltzmann constant. Using (4) and (5) this $k_{r}$ becomes

$$
k_{r}=\int_{E=0}^{\infty} \frac{\Re^{+}(E) \exp \left(-E / k_{B} T\right) d E}{h Q} .
$$


Integration by parts yields

$$
k_{r}=\frac{k T}{h} \int_{E \rightarrow 0}^{\infty} \frac{\exp \left(-E / k_{B} T\right) d \mathfrak{N}^{+}(E)}{Q},
$$

since $\mathfrak{T}^{+}(0)$ is zero; $k$ now denotes $k_{B}$.

The integral in (13), which is a Stieltjes integral when $\mathfrak{T}^{+}(E)$ is quantized, is the partition function of the activated complex (measured relative to the zero of energy) $Q^{+}\left(s^{+}\right)$,

$$
\begin{aligned}
Q^{+}\left(s^{+}\right) & =\sum_{E_{N}} \exp \left(-\frac{E_{N}+}{k_{B} T}\right) \\
& =\int_{E=0}^{\infty} \exp \left(-\frac{E}{k_{B} T}\right) d \mathfrak{N}^{+}(E) .
\end{aligned}
$$

Thus (13) becomes the (canonical) activated complex theory expression

$$
k_{r}=\frac{(k T / h) Q^{+}\left(s^{+}\right)}{Q}
$$

The local free energy for these states is $F^{+}\left(s^{+}\right)$,

$$
F^{+}\left(s^{+}\right)=-k T \ln Q^{+}\left(s^{+}\right) .
$$

At the point $s^{+}$of maximum local free energy we have

$$
\partial F^{+}\left(s^{+}\right) / \partial s^{+}=0,
$$

and thus, from (14) and (16), this point occurs at

$$
\int_{0}^{\infty} \frac{\partial}{\partial s} \frac{d \mathfrak{N}^{+}(E)}{d E} \exp \left(-\frac{E}{k_{B} T}\right) d E=0 \quad\left(\text { at } s=s^{+}\right) \text {. }
$$

It follows that the maximum free energy criterion is a Boltzmann-factor-weighted minimum state density criterion.

If a subset of the microcanonical ensemble had been used, say a subset of constant total angular momentum, or with some other variable held constant, the $k_{r}$ obtaining by multiplying in (1) by

$$
\exp \frac{\left(-E / k_{B} T\right) d E}{h Q},
$$

using an appropriately restricted $Q$, would have represented the rate constant for the corresponding subset in the canonical ensemble. The condition (17) would have been that derived for this restricted ensemble and so, once again, the two criteria for the position of $s^{+}$would have been similarly related.

\section{APPENDIX: EQUATIONS FOR NUMBER OF STATES ${ }^{18}$}

We collect here several formulas useful for computing $\Re^{+}\left(E^{+}\right)$and $\Re(E)$. Each of these quantities can be re- garded as convolutions for the corresponding quantities for rotations and vibrations when rotation-vibration interaction is neglected. For example, when $Q^{+}(\beta)$ ) the partition function of the activated complex $(\beta=$ $1 / k T)$, is the product of a vibrational factor $Q^{+}(\beta)$ and a classical rotational partition function, $A_{R}{ }^{+} / \beta^{q+1}$,

$$
Q^{+}(\beta)=\left(A_{R}^{+} / \beta^{q+1}\right) Q_{V}{ }^{+}(\beta),
$$

inversion via a Laplace transform and use of the convolution theorem yields

$\mathfrak{N}^{+}(E)=A_{R^{+}} \int_{0}^{E-V^{+}} \frac{\left(E-E_{v}{ }^{+}-V^{+}\right)^{q} N_{v}{ }^{+}\left(E_{v}{ }^{+}\right) d E_{v}{ }^{+}}{\Gamma(q+1)}$,

where $N_{v}^{+}\left(E_{v}^{+}\right)$is the number of vibrational states with energy equal to or less than $E_{v}{ }^{+}$. When a quantum expression is used for $\mathfrak{\Re}_{v}{ }^{+}\left(E_{v}{ }^{+}\right), \mathfrak{N}_{v}{ }^{+}\left(E_{v}\right)$ is zero for $E_{v}{ }^{+}$less than the zero-point energy $E_{0}{ }^{+}$of the activated complex and consists of a sum of delta functions centered at each energy level. A frequently used semiclassical expression for $\mathfrak{N}_{v}^{+}\left(E_{v}{ }^{+}\right)$(when the oscillators are harmonic) is

$$
\Re_{0}^{+}\left(E_{v}^{+}\right)=\frac{\left(E_{v}^{+}-E_{0}^{+}+a E_{0}^{+}\right)^{M}}{M !} \prod_{i=1}^{M} h \nu_{i}^{+},
$$

where $M$ is the number of vibrational degrees of freedom of the activated complex. $E_{v}{ }^{+}$is the total vibrational energy including the zero-point energy $E_{0}+$. The constant $a$ is unity in the classical limit. In a quantum treatment it equals unity at higher temperatures and is a known function of $E_{v}{ }^{+}$at lower temperatures.

The expression $\sum_{N}\left(k^{2} / \pi\right)$ is given by Eq. (7). The expression for $\mathfrak{N}\left(E_{N}\right)$ to be used there is identical with (A1), but with $V^{+}, A_{R}{ }^{+}, E_{v}{ }^{+}$, and $q$ replaced by the values appropriate to the pair of reactants. In this way Eqs. (8) and (9) are readily obtained.

A convenient collection of results for specific classical cases is also given by Morokuma, Eu, and Karplus in Ref. 4.

* Acknowledgment is made to the National Science Foundation and to the donors of the Petroleum Research Fund, administered by the American Chemical Society, for financial support.

$\dagger$ Present address: Department of Chemistry, University of Toronto, Toronto, Canada.

1 R. A. Marcus, J. Chem. Phys. 45, 2138 (1966).

2 (a) R. A. Marcus, J. Chem. Phys. 45, 2630 (1966); (b) 46, 959 (1967).

${ }^{3}$ R. A. Marcus, J. Chem. Phys. 53, 604 (1970).

4 K. Morokuma, B. C. Eu, and M. Karplus, J. Chem. Phys. 51,4193 (1969).

"G. W. Koeppl and M. Karplus "Comparison of 3D Trajectory and Transition-State Theory Reaction Cross Sections," J. Chem. Phys. (to be published) and K. Morokuma and M. Karplus, (to be published), cited in Ref. 6 .

${ }^{6} \mathrm{~K}$. Morokuma and M. Karplus, J. Chem. Phys. 54, 63 (1971).

${ }^{7}$ For example, S. Glasstone, K. J. Laidler, and H. Eyring, Theory of Rate Processes (McGraw-Hill, New York, 1940), p. 196. 
${ }^{8}$ D. L. Bunker and M. Pattengill, J. Chem. Phys. 48, 772 (1968).

"We shall use the form of "adiabatic" transition state theory described in R. A. Marcus, J. Chem. Phys. 43, 1598 (1965). In this approach we use adiabaticity only in the vicinity of the activated complex. In Ref. 10 (apart from references to R. A. M.) adiabaticity is assumed throughout.

${ }^{10}$ An adiabatic transition state theory is discussed by $M$. A. Eliason and J. O. Hirschfelder, J. Chem. Phys. 30, 1426 (1959); L. Hofacker, Z. Naturforsch. 18a, 607 (1963); R. A. Marcus, J. Chem. Phys. 41, 2614, 2624 (1964); M. S. Child, Discussions Faraday Soc. 44, 68 (1967).

11 Reference 1, Eq. (5).

12 For interesting discussions of the application of adiabatic transition state theory and related topics see D. G. Truhlar, J. Chem. Phys. 53, 2041 (1970); D. G. Truhlar and A.
Kupperman, ibid., 52, 3841 (1970); K. Morokuma and M. Karplus, Ref. 6.

${ }^{13}$ For example, when the reaction is one of free-radical recombination leading to a product in a singlet electronic state one might multiply the result by a factor of $\frac{1}{4}$, when $\frac{3}{4}$ of the competitive steps lead to a repulsive triplet state.

14 Reviewed in W. Frost and Z. Prasil, J. Chem. Phys. 51, 3006 (1969); 53, 3065 (1970).

${ }^{15}$ H. S. Johnston, Gas Phase Reaction Rate Theory (Ronald, New Vork, 1966).

${ }^{16}$ R. A. Marcus, J. Chem. Phys. 43, 2658 (1965).

${ }^{17} \mathrm{~J}$. C. Polanyi and W. H. Wong, J. Chem. Phys. (to be published).

18 Taken largely from Eqs. (28), (30), and (33) in Ref. 3. Extensive references to original studies are given there. Equation (A3) is that of Rabinovitch and co-workers (Ref. 19 in Ref. 3).

\title{
Nuclear Magnetic Resonance of the Aquated Proton. II. Chloroauric Acid Tetrahydrate. Phase Transitions and Molecular Motion*
}

\author{
Donald E. O'Reilly, E. M. Peterson, C. E. Scheie, $†$ and Jack M. Wrlliams \\ Argonne National Laboratory, Argonne, Illinois 60430
}

(Received 13 July 1971)

\begin{abstract}
Proton and deuterium nuclear magnetic resonance and chlorine-35 nuclear quadrupole resonance of chloroauric acid tetrahydrate, $\mathrm{H}_{5} \mathrm{O}_{2}{ }^{+} \mathrm{AuCl}_{4}-2 \mathrm{H}_{2} \mathrm{O}$ has been studied from 180 to $300^{\circ} \mathrm{K}$. Phase transitions are observed at $290^{\circ} \mathrm{K}$ (Solid I $\leftrightarrow$ Solid II) and at $218^{\circ} \mathrm{K}$ (Solid II $\leftrightarrow$ Solid III) in ordinary chloroauric acid tetrahydrate. In the deuterated compound the lower-phase transition shifts upward by $33^{\circ}$ to $252^{\circ} \mathrm{K}$; an upper-phase transition was not detected below $300^{\circ} \mathrm{K}$. The chlorine-35 NQR consists of two lines $(1: 1$ intensity ratio) above the Solid II-III phase transition and three lines (1:2:1 intensity ratio) below this transition. The proton spin-lattice relaxation time $T_{1}$ of $\mathrm{H}_{5} \mathrm{O}_{2}{ }^{+} \mathrm{AuCl}_{4}-\cdot 2 \mathrm{H}_{2} \mathrm{O}$ exhibits a minimum at $273^{\circ} \mathrm{K}$ which is assigned to the interbond jump motion of the bridging proton in the $\mathrm{H}_{6} \mathrm{O}_{2}{ }^{+}$ion. The activation energy for this process is $5.7 \mathrm{kcal} \mathrm{mole}^{-1}$ in Solid II and $11.7 \mathrm{kcal} \mathrm{mole}^{-1}$ in Solid III. Rotating frame relaxation times have minima at 200 and $233^{\circ} \mathrm{K}$ which are assigned to the interbond jump process and a proton exchange process, respectively. The proton exchange process involves rotation of the hydronium ion about the threefold axis and translational diffusion of water molecules between sites which differ in water molecule orientation by $90^{\circ}$. The deuteron resonance of a single crystal and polycrystalline $\mathrm{D}_{5} \mathrm{O}_{2}+\mathrm{AuCl}_{4}-\cdot 2 \mathrm{D}_{2} \mathrm{O}$ is interpreted in terms of the deuterium exchange process. This process has an activation energy of $10.4 \mathrm{kcal}^{\mathrm{mole}} \mathrm{e}^{-1}$ in Solid II.
\end{abstract}

\section{INTRODUCTION}

The diaquated proton $\mathrm{H}_{5} \mathrm{O}_{2}{ }^{+}$has been demonstrated to exist by $\mathrm{x}$-ray diffraction studies of $\mathrm{H}_{5} \mathrm{O}_{2}+\mathrm{Cl}^{- \text {, }}{ }^{\text {la }} \mathrm{H}_{5} \mathrm{O}_{2}+\mathrm{Cl}^{-} \cdot \mathrm{H}_{2} \mathrm{O}^{\text {,b }}$ and $\mathrm{H}_{5} \mathrm{O}_{2}+\cdot \mathrm{ClO}_{4}{ }^{-\mathrm{lc}}$ and also by neutron diffracton experiments on trans $\left[\mathrm{Co}(\mathrm{en}){ }_{2} \mathrm{Cl}_{2}{ }^{+}\right] \mathrm{Cl}^{-}\left(\mathrm{H}_{5} \mathrm{O}_{2}{ }^{+}\right) \mathrm{Cl}^{-},{ }^{2 \mathrm{a}}$ nitranilic acid hexahydrate, ${ }^{2 \mathrm{~b}}$ and chloroauric acid tetrahydrate, ${ }^{2 \mathrm{c}}$ $\mathrm{H}_{5} \mathrm{O}_{2}{ }^{+} \mathrm{AuCl}_{4}^{-} \cdot 2 \mathrm{H}_{2} \mathrm{O}$. Williams and Peterson ${ }^{2 c}$ showed that in $\mathrm{HAuCl}_{4} \cdot 4 \mathrm{H}_{2} \mathrm{O}$ the diaquated proton exists in a nonplanar, trans configuration with the bridging proton in off-center positions. From the neutron diffraction studies it was not concluded whether the disorder of the bridging protons is static or dynamic. Proton relaxation times $\left(T_{1}\right.$ and $\left.T_{1 \rho}\right)$ provide a means of evaluating the nature of the protonic disorder. Also of interest are the results of deuteron resonance studies on $\mathrm{DAuCl}_{4} \cdot 4 \mathrm{D}_{2} \mathrm{O}$ to lend support to the neutron diffraction studies.
Bateman, in thesis work, ${ }^{3}$ made limited studies of the chlorine nuclear quadrupole resonance (NQR) of $\mathrm{HAuCl}_{4} \cdot 4 \mathrm{H}_{2} \mathrm{O}$ and reported a phase transition at $219^{\circ} \mathrm{K}$. In the present work the chlorine NQR measurements are repeated, extended to higher temperatures, and recorded for the deuterated compound.

$A b$ initio self-consistent-field molecular-orbital calculations on the $\mathrm{H}_{5} \mathrm{O}_{2}{ }^{+}$species with various structures have been carried out by Kollman and Allen ${ }^{4}$ and by Newton and Ehrenson. ${ }^{5}$ Kollman and Allen found that an $s p^{2}$ configuration around the oxygens with staggered arrangement of protons was most stable. The minimum energy O-O distance found was $2.38 \AA$ for which distance the bridging proton potential has a single flat-bottomed minimum. At larger $\mathrm{O}-\mathrm{O}$ distances Kollman and Allen find a double minimum proton potential; in $\mathrm{HAuCl}_{4} \cdot 4 \mathrm{H}_{2} \mathrm{O}$ the $\mathrm{H}_{5} \mathrm{O}_{2}+\mathrm{O}-\mathrm{O}$ distance is $2.57 \pm 0.01 \AA .2 \mathrm{c}$

In the present work additional neutron diffraction 